\title{
On-farm Soil Health Assessment of Cover-cropping in Florida
}

\author{
Jehangir H. Bhadha ${ }^{1}$, Nan $\mathrm{Xu}^{2}$, Abul Rabbany ${ }^{3}$, Naba R. Amgain ${ }^{4}$, Jay Capasso ${ }^{5}$, Kevin Korus ${ }^{6}$ \& Stewart \\ Swanson $^{7}$ \\ ${ }^{1}$ Assistant Professor, University of Florida - Soil and Water Sciences Department, Belle Glade, Florida, USA \\ ${ }^{2}$ Graduate Student, University of Florida - Soil and Water Sciences Department, Belle Glade, Florida, USA \\ ${ }^{3}$ Chemist III, University of Florida - Everglades Research and Education Center, Belle Glade, Florida, USA \\ ${ }^{4}$ Postdoctoral Associate, University of Florida - Everglades Research and Education Center, Belle Glade, \\ Florida, USA \\ ${ }^{5}$ Extension Agent I (Columbia County) and Graduate Student, University of Florida - Soil and Water Sciences \\ Department, Belle Glade, Florida, USA \\ ${ }^{6}$ Extension Agent II (Alachua County), University of Florida - IFAS Extension, Gainesville, Florida, USA \\ ${ }^{7}$ Extension Agent IV (Hendry County), University of Florida - IFAS Extension, LaBelle, Florida, USA \\ Correspondence: Jehangir H. Bhadha, Assistant Professor, University of Florida - Soil and Water Sciences \\ Department, Belle Glade, Florida, USA. E-mail: jango@ufl.edu
}

Received: December 11, 2020 Accepted: January 20, 2021 Online Published: January 29, 2021

doi:10.5539/sar.v10n2p17

URL: https://doi.org/10.5539/sar.v10n2p17

\begin{abstract}
Conventional cropping systems on sandy soils require continuous application of large amounts of external nutrients and irrigation water yet remain vulnerable to loses of these inputs. Within the state of Florida, need exists to provide farmers with economically viable alternatives that harness ecological processes and improve soil health and biodiversity. Cover crops are proving to be vital in the development of soil health. As part of this study we conducted a comprehensive on-farm assessment involving nine collaborative growers (ten farms) across the state; with each individual farm following its unique cover-cropping practice. Our goal was to shadow their practice and determine its effect on soil health indicators such as soil $\mathrm{pH}$, bulk density (BD), maximum water holding capacity (MWHC), organic matter (OM), active carbon, cation exchange capacity, soil protein, Total Kjeldahl nitrogen (TKN), total phosphorus (TP), Mehlich-3 P (M3P) and potassium (M3K). Compared to fallow, soil OM, MWHC, and soil protein showed increases in cover crop fields for most farms, which presented a positive change towards building up soil health. Although soil TKN level was significantly decreased due to cover crops, soil protein level building up over time was the most positive change for soil health. M3K decreased in cover-crop fields, which indicated that supplementary $\mathrm{K}$ would be necessary prior to planting subsequent cash crops.
\end{abstract}

Keywords: cover crop, organic matter, active carbon, sandy soil, soil protein, soil health

\section{Introduction}

\subsection{Rational \& Justification}

Over the past century, farming and agriculture production in the United States has shifted from highly diversified low-input systems to highly specialized operations largely dependent on external non-renewable resources. Farm size has increased dramatically, along with an erosion of farm and crop diversity. Since 1900, the number of farms has fallen by 63 percent, while the average farm size has risen 67 percent (Dimitri 2005). This has resulted in agro-ecosystems more vulnerable to pressures from urbanization, climate change, and volatile global markets (Fischer et al., 2005). Need exists to provide farmers with economically viable alternatives that harness ecological processes which will improve soil health and crop diversity. With a production area of over 2 million acres and a crop value of almost 5 billion dollars annually, vegetable, fruit and field crop production comprise major agricultural activities in Florida. Most of the state's soils possess little organic matter $(<2 \%)$ and exhibit poor water and nutrient retention capacity, especially those experiencing regular disturbances through tillage and low inputs of organic matter (Bhadha et al., 2017a). Conventional cropping systems on such soils therefore require continuous application of large amounts of external nutrients and irrigation water yet remain vulnerable 
to large losses of these inputs.

Agronomic soils in Florida comprise of seven out of the twelve soil orders - Spodosols, Entisols, Ultisols, Alfisols, Histosols, Mollisols, and Inceptisols (Mylavarapu et al., 2019). Spatially the Spodosols are most extensively occurring soils in Florida, covering approximately $34,000 \mathrm{~km}^{2}$, whereas Inceptisols cover only about $4,047 \mathrm{~km}^{2}$ in the state. In general, the soils across the U.S. Southeastern coastal plains including Florida were mostly developed under forested land with abundant rainfall. The high precipitation leads to leaching of base cations such as potassium $(\mathrm{K})$, calcium $(\mathrm{Ca})$, magnesium $(\mathrm{Mg})$, and sodium $(\mathrm{Na})$, rendering the soils mostly acidic. The underlying bedrock is typically composed of sandy marine sediments north of Lake Okeechobee and calcareous limestone to the south. Shallow soil in the south of the state intermixed with calcareous material tends to exhibit higher $\mathrm{pH}$ in soils (Bhadha et al., 2020), and this can limit the bioavailability of micronutrients to crops (He et al., 2000; Fageria et al., 2002).

Cover crops and the practice of green manure are proving to be vital in the development of soil health (Snapp et al., 2005). Growing cover crops is perhaps the most valuable strategy we can adopt to feed our soil, build up its fertility and improve its structure with each passing season. Practical information about the physical, chemical and biological transformation of soils resulting from cover-cropping over a growing season is often lacking. Most existing information on cover-cropping and green manure practice performance comes from studies conducted in temperate, mid-western, fine-textured soils, the results of which may not apply to Florida with its sub-tropical/sub-temperate climate and sandy soil. It is not known if green manure can supply adequate nutrients if amount and timing of nutrient release do not match crop demand, especially for high nitrogen $(\mathrm{N})$ demanding vegetables. In addition, little to no information exists on physical transformation to soil properties, such as maximum water holding capacity and bulk density as a result of cover-cropping. It also remains unclear if long-term improvements in soil fertility can be achieved under Florida conditions.

\subsection{Objectives}

The purpose of this research is to evaluate the changes in soil health indicators as a function of cover-cropping. In order to test our objective, we identified growers across Florida that practiced cover-cropping on their farms over either summer or winter fallow periods. Once we had identified the growers, we collected soil samples before and after cover crops, to evaluate the changes in soil health indicators as a function of cover-cropping. Two specific objectives of this study were to: (i) quantify the changes in soil health indicators of cultivating cover crops on each farm over the fallow periods; (ii) evaluate the cover-cropping practices performance in Florida based on the results of all farms involved in this study.

\section{Materials and Methods}

\subsection{Study Sites and Cover Crops}

This study was conducted on ten agricultural farms (Figure 1) with sizes ranging from 25 to 400 acres across Florida including sugarcane (Saccharum officinarum), sweetcorn (Zea mays L.) and vegetable growers (Table 1). The climate of Florida ranges from humid subtropical in the north and central parts to tropical in the south. The average annual temperature of Florida is $21.2^{\circ} \mathrm{C}$, and average annual precipitation is $1,364 \mathrm{~mm}$ (The Southeast Regional Climate Center, 1895-2020 Data, https://sercc.com/climateinfo/monthly_seasonal). The soils in the study sites were mainly mineral soils including Spodosols, Entisols, Ultisols, and Alfisols (Table 1). Spodosols are characterized by intense leaching, with a low nutrient retention capacity, particularly phosphorus (P) retention. Entisols and Ultisols have a low cation exchange capacity (CEC) and a low level of base cations due to leaching. While Alfisols have relatively high CEC and base saturation, they are exposed to leaching in Florida from frequent rainfall events, thus management practices such as cover crops rotation with row crops are recommended to maintain soil fertility (Mylavarapu et al., 2019). Overall, common soil health constraints associated with these soils under Florida climate condition include relatively poor nutrient and water holding capacities, low soil organic matter content and high leaching potential (Bhadha et al., 2017b).

A number of cover crops are adapted in Florida (https://sfyl.ifas.ufl.edu/agriculture/cover-crops/). Annual cover crops are most commonly used, especially in intensive vegetable rotation systems. These cover crops can be either winter crops that are adapted to cooler season or summer crops that are adapted to hot season. Due to the climatic variability across the state from north to south, in North Florida the fallow period is usually winter months, while in South Florida summer months are usually fallow. The tropical climate in the southern portion of the state is suitable for growing tropical legumes and forages, while in the northern region, winter legumes and grains are often grown (Treadwell, Klassen \& Alligood, 2008). Summer cover crops are typically planted as a supplementary $\mathrm{N}$ source for the subsequent vegetable crops, while winter cover crops are grown as nutrient sinks, which scavenge the soils remaining nutrients. 
Since this was a comprehensive on-farm study with multiple collaborative growers across the state, each individual farm followed its unique cover-cropping practice. Our goal was to shadow their practice and determine its effect on soil health indicators. Different varieties of cover crops were grown on the farms in rotation with cash crops, either in summer or winter, or both summer and winter periods (Table 1). For example, the farm in Site 1 usually grows vegetables, and it performed both summer and winter cover-cropping practice during the experimental period (2018-2019); growing different mixes of legumes and grains as described in Table 1. The farm in Site 5 planted cover crops in summer fallow period (2018), including buckwheat (Fagopyrum esculentum) followed by cowpea (Vigna unguiculata)/sunn hemp (Crotalaria juncea) mix in the same experimental fields. The farm in Site 7 performed summer cover-cropping practice in both 2018 and 2019, with planting cowpea only in several experimental fields while cowpea/sudan grass (Sorghum $\times$ drummondii) mix in another experimental fields in 2018 , and planting cowpea/sunn hemp mix in all experimental fields in 2019.

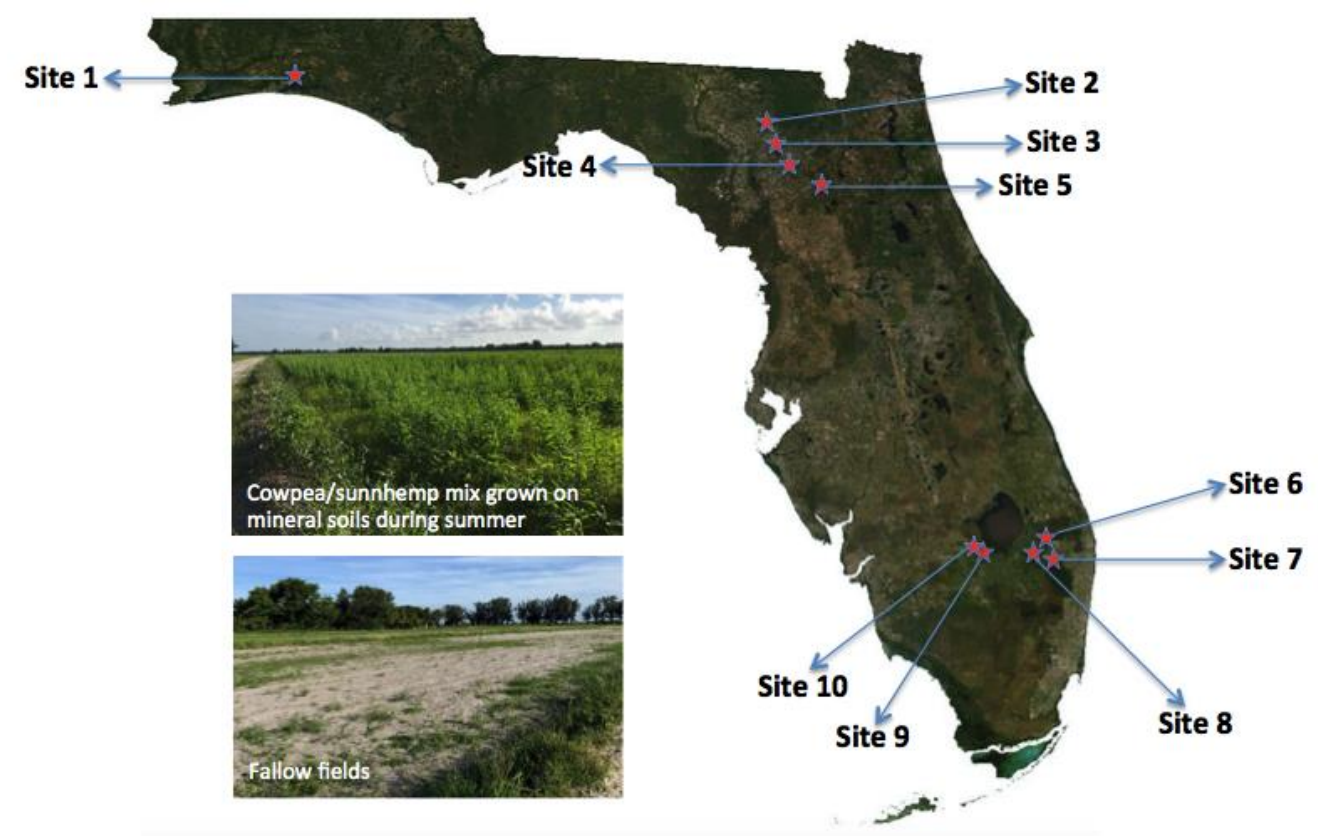

Figure 1. Map of study sites (farms) located in Florida. The farm locations are indicated by stars. Two insets (cover-cropping practice vs. fallow fields) are taken on Site 8 
Table 1. Farm Type, Location, Soil Order, Cover Crops, and Cover Crops Performing Dates in each Study Site

\begin{tabular}{|c|c|c|c|c|c|}
\hline Site & Farm Type & Location & & Cover Crops & Cover Crops \\
\hline & & & Order & & Performing Dates \\
\hline 1 & Vegetables & $\begin{array}{l}\text { Walton } \\
\text { County }\end{array}$ & Ultisols & $\begin{array}{l}\text { Alfalfa (Medicago sativa), Buckwheat, Mustard seeds } \\
\text { (Brassica nigra), Oats (Avena sativa), Peas (Pisum sativum) } \\
\text { Barley (Hordeum vulgare), Cereal rye (Secale cereale), } \\
\text { Crimson clover (Trifolium incarnatum), Faba Beans (Vicia } \\
\text { faba), Flax (Linum usitatissimum), Lentil (Lens culinaris), } \\
\text { Oats, Peas, Triticale ( } X \text { Triticosecale), Radish (Raphanus } \\
\text { sativus), Rye, Safflower (Carthamus tinctorius), Vetch } \\
\text { (Vicia), Wheat (Triticum), White clover (Trifolium repens) }\end{array}$ & $\begin{array}{l}\text { Summer: May-August } \\
2018 \\
\text { Winter: December } \\
\text { 2018-April } 2019\end{array}$ \\
\hline 2 & Pasture & $\begin{array}{l}\text { Columbia } \\
\text { County }\end{array}$ & Ultisols & Oats \& Rye mix & $\begin{array}{l}\text { Winter: December } \\
\text { 2018-April } 2019\end{array}$ \\
\hline 3 & Peanut & $\begin{array}{l}\text { Columbia } \\
\text { County }\end{array}$ & Ultisols & Oats & $\begin{array}{l}\text { Winter: December } \\
\text { 2018-April } 2019\end{array}$ \\
\hline 4 & Hay & $\begin{array}{l}\text { Columbia } \\
\text { County }\end{array}$ & Ultisols & Oats & $\begin{array}{l}\text { Winter: December } \\
\text { 2018-April } 2019\end{array}$ \\
\hline 5 & $\begin{array}{l}\text { Vegetables, } \\
\text { fruits, flowers }\end{array}$ & $\begin{array}{l}\text { Alachua } \\
\text { County }\end{array}$ & Entisols & Summer: Buckwheat, Cowpea \& Sunn hemp mix & $\begin{array}{l}\text { Summer: May-August } \\
2018\end{array}$ \\
\hline 6 & Vegetables & $\begin{array}{l}\text { Palm } \\
\text { Beach } \\
\text { County }\end{array}$ & Alfisols & Sunn hemp & $\begin{array}{l}\text { Summer: May-August } \\
2018\end{array}$ \\
\hline 7 & $\begin{array}{l}\text { Organic } \\
\text { Vegetables }\end{array}$ & $\begin{array}{l}\text { Palm } \\
\text { Beach } \\
\text { County }\end{array}$ & Alfisols & $\begin{array}{l}\text { Cowpea, Cowpea \& Sudan grass mix } \\
\text { Cowpea \& Sunn hemp mix }\end{array}$ & $\begin{array}{l}\text { Summer: May-August } \\
2018 \\
\text { Summer: May-August } \\
2019\end{array}$ \\
\hline 8 & Vegetables & $\begin{array}{l}\text { Palm } \\
\text { Beach } \\
\text { County }\end{array}$ & Alfisols & $\begin{array}{l}\text { Cowpea \& Sunn hemp mix } \\
\text { Cowpea \& Sunn hemp mix }\end{array}$ & $\begin{array}{l}\text { Summer: May-August } \\
2018 \\
\text { Summer: May-August } \\
2019\end{array}$ \\
\hline 9 & Sugarcane & $\begin{array}{l}\text { Hendry } \\
\text { County }\end{array}$ & Spodosols & Sunn hemp & $\begin{array}{l}\text { Summer: May-August } \\
2018\end{array}$ \\
\hline 10 & Sugarcane & $\begin{array}{l}\text { Hendry } \\
\text { County }\end{array}$ & Spodosols & Sunn hemp & $\begin{array}{l}\text { Summer: May-August } \\
2019\end{array}$ \\
\hline
\end{tabular}

\subsection{Soil Health Assessment Framework and Analytical Methods}

Figure 2 outlines the soil health assessment framework that was followed in this study to determine the changes in soil health indicators as a function of cover-cropping. This was a 1.5-year study from April 2018 to August 2019, with planting cover crops or fallow during May-August 2018/2019 and December 2018-April 2019 periods between cash crops. Six experimental fields were selected on each study site for performing cover-cropping practice and one composite soil sample was collected from the top $15 \mathrm{~cm}$ from each field. On Sites 1, 7, 8, and 9 one more composite soil sample was collected as control from an additional experimental field where cover crops were not grown. A composite soil sample comprised of mixing ten soil subsamples was collected along a transect within individual fields. The soil samples were collected twice from each field, just before planting cover crops (pre) and right after cover crops termination (post), approximately 4 or 5 months apart. All soil samples were collected using 1-gallon Ziploc bags and transported to the Soil, Water, and Nutrient Management Laboratory at the University of Florida - Everglades Research and Education Center, Belle Glade for soil health evaluation. We evaluated cover crops performance on soil health for two years, in which year 1 and year 2 was treated as a variable since not all experimental sites cultivated cover crops in the second year and cover crops species were changed as well (Table 2). 
Table 2. Number of composite soil samples collected per site per year

\begin{tabular}{lllll}
\hline \multicolumn{5}{c}{ Numbers of Composite Soil Samples Collected } \\
\hline \multicolumn{3}{c}{ Year 1 } & \multicolumn{3}{c}{ Year 2 } \\
\hline Site & Control & Cover Crops & Control & Cover Crops \\
\hline 1 & 1 & 6 & 0 & 0 \\
2 & 0 & 6 & 0 & 0 \\
3 & 0 & 6 & 0 & 0 \\
4 & 0 & 6 & 0 & 0 \\
5 & 0 & 6 & 0 & 0 \\
6 & 0 & 6 & 0 & 0 \\
7 & 1 & 6 & 1 & 7 \\
8 & 2 & 4 & 2 & 4 \\
9 & 1 & 6 & 0 & 0 \\
10 & 0 & 0 & 1 & 6 \\
\hline
\end{tabular}

Pre (within one week prior to cover crops being planted) and post (within one week of cover crops being terminated) soil samples were air-dried, passed through 2-mm sieve and analyzed for physical, chemical, and biological soil health indicators as listed in Figure 2. Bulk density (BD) was determined by measuring soil mass in a known core volume. Maximum water holding capacity (MWHC) was determined using modified method described by Jenkinson and Powlson (1976) by measuring the amount of water retained in soil after saturation. Cation exchange capacity (CEC) was determined based on exchangeable ammonium using natural ammonium acetate exchange method (Sumner and Miller, 1996). Soil pH was determined with a 1:2 soil to water ratio (v/v) using Accumet AB250 pH meter. Extractable phosphorus and potassium (M3P and M3K) were determined using Mehlich 3 extraction method analyzed with Agilent 5110 inductively coupled plasma-optical emission spectrometer (ICP-OES) (Santa Clara CA). Total phosphorus (TP) was determined by ashing technique followed by extraction with $6 \mathrm{M} \mathrm{HCl}$ and analyzed with ICP. Total Kjeldahl nitrogen (TKN) was analyzed by digestion followed by colorimetric determination (EPA method 351.2). Active Carbon (C) was determined by quantifying potassium permanganate $\left(\mathrm{KMnO}_{4}\right)$ oxidizable carbon with a spectrophotometer (Schindelbeck, B.N. Moebius-Clune, D.J. Moebius-Clune, Kurtz, \& van Es, 2016). Soil organic matter (OM) content was calculated based on loss on ignition (LOI) method. Soil protein was determined using sodium citrate extraction method followed by colorimetric BCA determination (Schindelbeck, B.N. Moebius-Clune, D.J. Moebius-Clune, Kurtz, \& van Es, 2016).

\subsection{Statistical Analysis}

The differences in variations of soil health indicators of the pre and post soil samples between cover crops and fallow fields were compared by performing one-way ANOVA analysis using RStudio (RStudio, Inc) statistical software. When F-tests/ANOVA showed statistical significance, the Tukey's Test $(\mathrm{P}<0.05)$ was used to separate means for the different treatments.
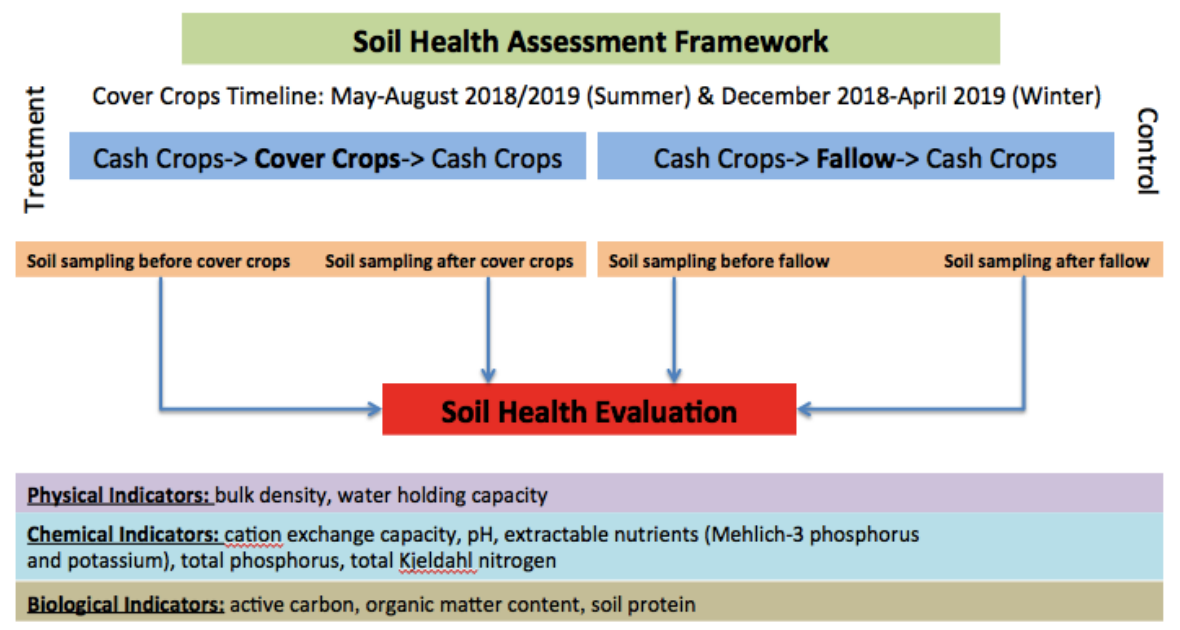

Figure 2. Soil Health Assessment Framework for this study 


\section{Results}

\subsection{Effects of Cover Crops on Each Individual Farm}

The farm in Site 1 utilized both summer and winter cover-cropping practice with various mixes of legumes and grains (Table 1) during the experimental period, from May to August 2018 and December 2018 to April 2019. Usually this farm grows vegetables as cash crops, but no vegetables were grown after summer cover crops termination between September and November 2018. Compared to fallow, cover-cropping practice performed in both seasons stabilized soil BD since there were no changes among the three sampling dates while BD changed in fallow plots (Table 3). Summer cover-cropping practice increased MWHC by $6.24 \%$, OM by $4 \%$, TKN by $21.84 \%$, and total $\mathrm{P}$ by $10.18 \%$ while these soil parameters decreased in fallow plots over the same time. No obvious effects were observed on soil $\mathrm{pH}$, active $\mathrm{C}, \mathrm{CEC}, \mathrm{M} 3 \mathrm{P}$, and soil protein due to cover-cropping practice since the percentage change trend was similar with fallow plots over the experimental period. However, cover-cropping practice had a lower percentage change in soil $\mathrm{pH}$ and active $\mathrm{C}$ while a higher percentage change in CEC, M3P and soil protein. There was a reduction in M3K when cover crops were planted compared to fallow.

Table 3. Soil Parameters Changes of Cover-Cropping Practices in May-August 2018 and December 2018-April 2019 on Site 1 (mean and standard deviation) (+) increase, (-) decrease, NA no change

\begin{tabular}{|c|c|c|c|c|c|c|c|c|}
\hline \multirow[t]{2}{*}{ Soil Parameters } & \multicolumn{2}{|c|}{$\begin{array}{l}\text { Mean of Pre Soil } \\
\text { Samples (Summer) }\end{array}$} & \multicolumn{2}{|c|}{$\begin{array}{l}\text { Mean of Post Soil } \\
\text { Samples (Summer) }\end{array}$} & \multicolumn{2}{|c|}{$\begin{array}{l}\text { Mean of Post } \\
\text { Soil Samples (Winter) }\end{array}$} & \multicolumn{2}{|l|}{$\%$ Change } \\
\hline & Fallow & $\begin{array}{l}\text { Cover } \\
\text { Crops } \\
\end{array}$ & Fallow & $\begin{array}{l}\text { Cover } \\
\text { Crops } \\
\end{array}$ & Fallow & $\begin{array}{l}\text { Cover } \\
\text { Crops } \\
\end{array}$ & Fallow & Cover Crops \\
\hline BD $\left(\mathrm{g} \mathrm{cm}^{-3}\right)$ & 1.35 & $1.35 \pm 0.02$ & 1.38 & $1.35 \pm 0.04$ & 1.36 & $1.35 \pm 0.05$ & $2.22(+) ; 1.45(-)$ & NA \\
\hline MWHC (\%) & 52.08 & $47.41 \pm 3.59$ & 52.04 & $50.37 \pm 2.13$ & 48.04 & $47.71 \pm 1.51$ & $0.08(-) ; 7.69(-)$ & $6.24(+) ; 5.28(-)$ \\
\hline $\mathbf{p H}$ & 6.09 & $6.26 \pm 0.28$ & 6.69 & $6.53 \pm 0.2$ & 6.38 & $6.43 \pm 0.16$ & $9.85(+) ; 4.63(-)$ & $4.31(+) ; 1.53(-)$ \\
\hline OM (\%) & 2.8 & $2.5 \pm 0.28$ & 2.4 & $2.6 \pm 0.29$ & 1.8 & $2.36 \pm 0.32$ & $14.29(-) ; 25(-)$ & $4(+) ; 9.23(-)$ \\
\hline $\begin{array}{l}\text { Active C } \\
\left(\mathrm{mg} \mathrm{kg}^{-1}\right)\end{array}$ & 1158 & $1185 \pm 19$ & 361.4 & $415.7 \pm 55$ & 921.6 & $745.9 \pm 106.8$ & $68.78(-) ; 155(+)$ & $64.93(-) ; 79.45(+)$ \\
\hline $\begin{array}{l}\text { CEC } \\
\left(\mathrm{cmolc} \mathrm{kg}^{-1}\right)\end{array}$ & 2.47 & $3.45 \pm 0.58$ & 2.37 & $2.54 \pm 0.65$ & 2.6 & $3.44 \pm 0.63$ & $4.05(-) ; 9.7(+)$ & $26.38(-) ; 35.43(+)$ \\
\hline TKN (mg kg-1) & 626.8 & $651 \pm 107$ & 620.5 & $793.1 \pm 125.7$ & 515.3 & $686.4 \pm 85.8$ & $1(-) ; 16.95(-)$ & $21.84(+) ; 13.46(-)$ \\
\hline $\begin{array}{l}\text { Total } \\
\left.\mathrm{kg}^{-1}\right)\end{array} \quad$ (mg & 192.8 & $194.8 \pm 28.3$ & 170.8 & $214.7 \pm 46.2$ & 147 & $203.3 \pm 39.6$ & $11.41(-) ; 13.91(-)$ & $10.18(+) ; 5.32(-)$ \\
\hline $\operatorname{M3P}\left(\mathrm{mg} \mathrm{kg}^{-1}\right)$ & 92.8 & $136.7 \pm 32.3$ & 84.5 & $107.6 \pm 17.8$ & 87.6 & $116.7 \pm 24.6$ & 8.94(-); 3.67(+) & $21.26(-) ; 8.43(+)$ \\
\hline $\operatorname{M3K}\left(\mathrm{mg} \mathrm{kg}^{-1}\right)$ & 43.8 & $98.23 \pm 26$ & 49.6 & $51 \pm 18.4$ & 4.62 & $3.7 \pm 2.9$ & $13.24(+) ; 90.69(-)$ & $48.08(-) ; 92.75(-)$ \\
\hline $\begin{array}{l}\text { Soil Protein } \\
\left(\mathrm{mg} \mathrm{kg}^{-1}\right)\end{array}$ & 247.9 & $283.6 \pm 29.6$ & 290.3 & $344.9 \pm 63.1$ & 245.8 & $291.7 \pm 42.8$ & $17.1(+) ; 15.32(-)$ & $21.64(+) ; 15.44(-)$ \\
\hline
\end{tabular}

The farm in Site 2 utilized winter cover-cropping practice with oats and rye mix that was periodically grazed by cattle from December 2018 to April 2019. Compared to pre soil samples, there was a decrease in BD, MWHC, $\mathrm{pH}$, TKN, soil protein, and M3K of post soil samples (Table 4). However, cover crops increased OM, active C, CEC, total P, and M3P. Particularly active C of post soil samples were increased by $108.2 \%$ compared to pre soil samples.

Table 4. Soil Parameters Changes of The First Year Cover-Cropping Practices in December 2018-April 2019 on Site 2 (mean and standard deviation) (+) increase, (-) decrease

\begin{tabular}{llll}
\hline Soil Parameters & Mean of Pre Soil Samples & Mean of Post Soil Samples & \% Change \\
\hline BD $\left(\mathbf{g ~ c m}^{-\mathbf{3}}\right)$ & Cover Crops & Cover Crops & Cover Crops \\
& $1.35 \pm 0.02$ & $1.33 \pm 0.04$ & $1.48(-)$ \\
MWHC $(\%)$ & $47.71 \pm 1.97$ & $46.37 \pm 2.66$ & $2.81(-)$ \\
pH & $6.35 \pm 0.05$ & $6.15 \pm 0.07$ & $3.15(-)$ \\
OM $(\%)$ & $2.77 \pm 0.15$ & $3.13 \pm 0.83$ & $13(+)$ \\
Active C $\left(\mathbf{m g ~ k g}^{-1}\right)$ & $193.2 \pm 13.7$ & $402.2 \pm 72.1$ & $108.2(+)$ \\
CEC $\left(\mathbf{c m o l c ~ k g}^{-1}\right)$ & $4.49 \pm 0.19$ & $5.02 \pm 1.63$ & $11.80(+)$ \\
TKN $\left(\mathbf{m g ~ k g}^{-1}\right)$ & $792.9 \pm 57$ & $711.4 \pm 78$ & $10.27(-)$ \\
Total P $\left.\mathbf{~} \mathbf{m g ~ k g}^{-1}\right)$ & $959 \pm 45$ & $1252 \pm 644$ & $30.57(+)$ \\
M3P $\left(\mathbf{m g ~ k g}^{-1}\right)$ & $282.4 \pm 10.7$ & $292.5 \pm 78.4$ & $3.6(+)$ \\
M3K $\left(\mathbf{m g ~ k g}^{-1}\right)$ & $92.66 \pm 3.83$ & $66.05 \pm 29.9$ & $28.72(-)$ \\
Soil Protein $\left(\mathbf{m g ~ k g}^{-\mathbf{1}}\right)$ & $287.6 \pm 20$ & $261 \pm 37$ & $9.24(-)$ \\
\hline
\end{tabular}


The farm in Site 3 performed winter cover-cropping practice with oats from December 2018 to April 2019. Compared to pre soil samples, soil BD and active $\mathrm{C}$ was increased in post soil samples (Table 5) while the other soil parameters were decreased. Active $\mathrm{C}$ showed the highest percentage increase $(85.81 \%)$ while M3K presented the highest decease $(68.93 \%)$.

Table 5. Soil Parameters Changes of The First Year Cover-Cropping Practices in December 2018-April 2019 on Site 3 (mean and standard deviation) (+) increase, (-) decrease

\begin{tabular}{llll}
\hline Soil Parameters & Mean of Pre Soil Samples & Mean of Post Soil Samples & \% Change \\
\hline BD $\left(\mathbf{g ~ c m}^{-\mathbf{3}}\right)$ & $1.26 \pm 0.02$ & $1.39 \pm 0.05$ & $10.32(+)$ \\
MWHC $(\boldsymbol{\%})$ & $52.71 \pm 1.63$ & $43.04 \pm 3.74$ & $18.35(-)$ \\
pH & $6.26 \pm 0.13$ & $6.12 \pm 0.14$ & $2.24(-)$ \\
OM $(\%)$ & $3.46 \pm 0.1$ & $2.66 \pm 0.74$ & $23.12(-)$ \\
Active C $\left(\mathbf{m g ~ k g}^{-\mathbf{1}}\right)$ & $174.2 \pm 11.2$ & $323.8 \pm 66$ & $85.81(+)$ \\
CEC $\left(\mathbf{c m o l c ~ k g}^{-1}\right)$ & $4.96 \pm 0.2$ & $4.27 \pm 1.27$ & $13.91(-)$ \\
TKN $\left(\mathbf{m g ~ k g}^{-1}\right)$ & $914.3 \pm 49.1$ & $547.5 \pm 175.2$ & $40.12(-)$ \\
Total P $\left(\mathbf{m g ~ k g}^{-\mathbf{1}}\right)$ & $146.4 \pm 3.9$ & $115.8 \pm 34.8$ & $20.95(-)$ \\
M3P $\left(\mathbf{m g ~ k g}^{-1}\right)$ & $48.5 \pm 3.75$ & $41.43 \pm 15.8$ & $14.58(-)$ \\
M3K $\left(\mathbf{m g ~ k g}^{-1}\right)$ & $55.19 \pm 5.65$ & $17.15 \pm 6.21$ & $68.93(-)$ \\
Soil Protein $\left(\mathbf{m g ~ k g}^{-\mathbf{1}}\right)$ & $344.9 \pm 48.9$ & $228.5 \pm 56.4$ & $33.74(-)$ \\
\hline
\end{tabular}

The farm in Site 4 performed winter cover-cropping practice with oats from December 2018 to April 2019. Compared to pre soil samples, there was an increase in soil BD, active C, total P, M3P, and M3K of post soil samples while the other soil properties were decreased (Table 6). M3K showed the highest increase, which is approximately $188.52 \%$. In addition, active $\mathrm{C}$, total $\mathrm{P}$, and M3P showed more than $50 \%$ increase, which was $86.61 \%, 66.27 \%$, and $52.77 \%$, respectively.

Table 6. Soil Parameters Changes of The First Year Cover-Cropping Practices in December 2018-April 2019 on Site 4 (mean and standard deviation) (+) increase, (-) decrease

\begin{tabular}{llll}
\hline Soil Parameters & Mean of Pre Soil Samples & Mean of Post Soil Samples & \% Change \\
\hline BD $\left(\mathbf{g ~ c m}^{-\mathbf{3}}\right)$ & $1.34 \pm 0.02$ & $1.46 \pm 0.03$ & $8.96(+)$ \\
MWHC $(\boldsymbol{\%})$ & $47.37 \pm 1.63$ & $40.04 \pm 2.19$ & $15.47(-)$ \\
pH & $6.09 \pm 0.05$ & $5.96 \pm 0.29$ & $2.13(-)$ \\
OM $(\%)$ & $2.8 \pm 0.18$ & $2.66 \pm 0.33$ & $5(-)$ \\
Active C $\left(\mathbf{m g ~ k g}^{-\mathbf{1}}\right)$ & $222.2 \pm 12.2$ & $414.7 \pm 47.4$ & $86.61(+)$ \\
CEC $\left(\mathbf{c m o l c ~ k g}^{-1}\right)$ & $4.43 \pm 0.71$ & $4.19 \pm 0.43$ & $5.42(-)$ \\
TKN $\left(\mathbf{m g ~ k g}^{-\mathbf{1}}\right)$ & $812.2 \pm 66.8$ & $653.7 \pm 130.4$ & $19.52(-)$ \\
Total P $\left(\mathbf{m g ~ k g}^{-\mathbf{1}}\right)$ & $416.5 \pm 19.1$ & $692.5 \pm 151.1$ & $66.27(+)$ \\
M3P $\left(\mathbf{m g ~ k g}^{-1}\right)$ & $251.9 \pm 36.1$ & $384.8 \pm 50.9$ & $52.77(+)$ \\
M3K $\left(\mathbf{m g ~ k g}^{-\mathbf{1}}\right)$ & $8.36 \pm 1.78$ & $24.12 \pm 7.43$ & $188.52(+)$ \\
Soil Protein $\left(\mathbf{m g ~ k g}^{-\mathbf{1}}\right)$ & $313 \pm 29.2$ & $290.9 \pm 29.1$ & $7.04(-)$ \\
\hline
\end{tabular}

The farm in Site 5 performed summer cover-cropping practice with buckwheat followed by cowpea and sunn hemp mix from May to August 2018. Compared to pre soil samples, there was a decrease in soil BD, pH, active $\mathrm{C}$, and M3K of post soil samples while the other soil parameters were increased (Table 7). Particularly a relatively higher decrease $(49.06 \%)$ was observed in active C of post soil samples compared to other soil properties. 
Table 7. Soil Parameters Changes of The First Year Cover-Cropping Practices in May-August 2018 on Site 5 (mean and standard deviation) (+) increase, (-) decrease

\begin{tabular}{llll}
\hline Soil Parameters & Mean of Pre Soil Samples & Mean of Post Soil Samples & \% Change \\
\hline BD $\left(\mathbf{g ~ c m}^{-\mathbf{3}}\right)$ & $1.37 \pm 0.05$ & $1.29 \pm 0.08$ & $5.84(-)$ \\
MWHC (\%) & $49.41 \pm 6.53$ & $51.71 \pm 5.72$ & $4.65(+)$ \\
pH & $7.61 \pm 0.19$ & $7.29 \pm 0.15$ & $4.2(-)$ \\
OM $(\%)$ & $4.33 \pm 0.71$ & $4.77 \pm 0.71$ & $10.16(+)$ \\
Active C $\left(\mathbf{m g ~ k g}^{-\mathbf{1}}\right)$ & $1253 \pm 13$ & $638.4 \pm 66.7$ & $49.06(-)$ \\
CEC $\left(\mathbf{c m o l c ~ k g}^{-1}\right)$ & $6.31 \pm 1.48$ & $7.65 \pm 1.6$ & $21.24(+)$ \\
TKN $\left(\mathbf{m g ~ k g}^{-1}\right)$ & $1219 \pm 184$ & $1389 \pm 170$ & $13.96(+)$ \\
Total P $\left(\mathbf{m g ~ k g ~}^{-1}\right)$ & $3373 \pm 1286$ & $4106 \pm 1407$ & $21.74(+)$ \\
M3P $\left(\mathbf{m g ~ k g}^{-1}\right)$ & $957 \pm 179.2$ & $1073 \pm 199$ & $12.1(+)$ \\
M3K $\left(\mathbf{m g ~ k g}^{-1}\right)$ & $162.7 \pm 88.6$ & $158.4 \pm 91.1$ & $2.37(-)$ \\
Soil Protein $\left(\mathbf{m g ~ k g}^{-1}\right)$ & $314.5 \pm 29.9$ & $359.9 \pm 27$ & $14.43(+)$ \\
\hline
\end{tabular}

The farm in Site 6 performed summer cover-cropping practice with sunn hemp from May to August 2018. Compared to pre soil samples, there was an increase in MWHC, CEC, TKN, M3P, and soil protein of post soil samples while the other soil properties were decreased (Table 8). Particularly active $\mathrm{C}$ and M3K showed a relatively higher decrease compared to other parameters, which was $77.25 \%$ and $68.04 \%$, respectively.

Table 8. Soil Parameters Changes of The First Year Cover-Cropping Practices in May-August 2018 on Site 6 (mean and standard deviation) (+) increase, (-) decrease

\begin{tabular}{llll}
\hline Soil Parameters & Mean of Pre Soil Samples & Mean of Post Soil Samples & \% Change \\
\hline BD $\left(\mathbf{g ~ c m}^{-\mathbf{3}}\right)$ & $1.44 \pm 0.04$ & $1.42 \pm 0.05$ & $1.39(-)$ \\
MWHC $(\boldsymbol{\%})$ & $38.75 \pm 2.07$ & $42.37 \pm 1.97$ & $9.34(+)$ \\
pH & $7.73 \pm 0.55$ & $7.27 \pm 0.23$ & $5.95(-)$ \\
OM $(\%)$ & $1.43 \pm 0.27$ & $1.03 \pm 0.41$ & $27.97(-)$ \\
Active C $\left(\mathbf{m g ~ k g}^{-\mathbf{1}}\right)$ & $1176 \pm 17$ & $267.6 \pm 63.3$ & $77.25(-)$ \\
CEC $\left(\mathbf{c m o l c ~ k g}^{-1}\right)$ & $1.47 \pm 0.23$ & $1.62 \pm 0.51$ & $10.2(+)$ \\
TKN $\left(\mathbf{m g ~ k g}^{-\mathbf{1}}\right)$ & $390.9 \pm 99.5$ & $453.1 \pm 161.7$ & $15.9(+)$ \\
Total P $\left(\mathbf{m g ~ k g}^{-\mathbf{1}}\right)$ & $204 \pm 41.1$ & $197.4 \pm 54.2$ & $3.23(-)$ \\
M3P $\left(\mathbf{m g ~ k g} \mathbf{~ g}^{-1}\right)$ & $119.3 \pm 16$ & $131.2 \pm 20.9$ & $9.98(+)$ \\
M3K $\left(\mathbf{m g ~ k g}^{-\mathbf{1}}\right)$ & $39.95 \pm 9.48$ & $12.77 \pm 10.54$ & $68.04(-)$ \\
Soil Protein $\left(\mathbf{m g ~ k g}^{-\mathbf{1}}\right)$ & $126.6 \pm 23.1$ & $165.9 \pm 38.8$ & $31.05(+)$ \\
\hline
\end{tabular}

The farm in Site 7 performed summer cover-cropping practice in both 2018 and 2019, planting cowpea only and cowpea/sudan grass mix in different experimental plots in 2018, and planting cowpea/sunn hemp mix in all experimental fields in 2019. Compared to fallow, there was a decrease in OM, TKN, total P, and soil protein in 2018 by performing cover-cropping practice (Table 9a). Cover crops also showed a higher percentage decrease in active $\mathrm{C}$ and CEC than fallow in 2018. Although total $\mathrm{P}$ was decreased, M3P was increased by $41.88 \%$ due to cover-cropping practice in 2018. However, results showed differently over summer 2019. All soil parameters except $\mathrm{BD}$ and $\mathrm{pH}$ presented a similar percentage change trend between cover-cropping practice and fallow (Table 9b). MWHC, Active C, and TKN showed a higher percentage decrease in cover crops than in fallow. 
Table 9a. Soil Parameters Changes of the First Year Cover-Cropping Practices in May-August 2018 on Site 7 (+) increase, (-) decrease, NA no change

\begin{tabular}{|c|c|c|c|c|c|c|}
\hline \multirow[t]{2}{*}{ Soil Parameters } & \multicolumn{2}{|c|}{ Mean of Pre Soil Samples } & \multicolumn{2}{|c|}{ Mean of Post Soil Samples } & \multicolumn{2}{|c|}{ \% Change } \\
\hline & Fallow & Cover Crops & Fallow & Cover Crops & Fallow & Cover Crops \\
\hline BD $\left(\mathrm{g} \mathrm{cm}^{-3}\right)$ & $1.06 \pm 0.06$ & $1.05 \pm 0.09$ & $1.06 \pm 0.06$ & $1.11 \pm 0.08$ & NA & $5.71(+)$ \\
\hline MWHC (\%) & $62.75 \pm 10.07$ & $62.08 \pm 5.16$ & $04 \pm 1$ & $75.29 \pm 6.18$ & $25.97(+)$ & $21.28(+)$ \\
\hline pH & $8.56 \pm 0.22$ & $8.5 \pm 0.21$ & $7.63 \pm 0.26$ & $7.78 \pm 0.36$ & $10.86(-)$ & $8.47(-)$ \\
\hline OM $(\%)$ & $.13 \pm 1.03$ & $12.8 \pm 3.75$ & $10.71 \pm 0.42$ & $9.47 \pm 1.62$ & $5.73(+)$ & $26.02(-)$ \\
\hline Active C (mg kg $\left.{ }^{-1}\right)$ & $1377 \pm 4.32$ & $1381 \pm 35$ & $1208 \pm 62$ & $1140 \pm 116$ & $12.27(-)$ & $17.44(-)$ \\
\hline CEC (cmolc kg-1) & $13.79 \pm 2.99$ & $14.79 \pm 4.5$ & $13.44 \pm 1.99$ & $10.66 \pm 1.45$ & $2.54(-)$ & $27.92(-)$ \\
\hline TKN (mg kg $\left.{ }^{-1}\right)$ & $3962 \pm 723$ & $5575 \pm 1148$ & $6357 \pm 1478$ & $4188 \pm 520$ & $60.45(+)$ & $24.88(-)$ \\
\hline Total $\mathbf{P}\left(\mathrm{mg} \mathrm{kg}^{-1}\right)$ & $787 \pm 82$ & $946 \pm 334$ & $934 \pm 61$ & $610 \pm 141$ & $18.69(+)$ & $35.52(-)$ \\
\hline $\operatorname{M3P}\left(\mathrm{mg} \mathrm{kg}^{-1}\right)$ & $187.4 \pm 2.8$ & $133.3 \pm 54.7$ & $130.6 \pm 23$ & $189.1 \pm 93.8$ & $30.31(-)$ & $41.88(+)$ \\
\hline $\operatorname{M3K}\left(\mathrm{mg} \mathrm{kg}^{-1}\right)$ & $175.8 \pm 190.7$ & $396.4 \pm 318.3$ & $51.13 \pm 7.18$ & $122.7 \pm 71.3$ & $70.91(-)$ & 69.04(-) \\
\hline Soil Protein $\left(\mathrm{mg} \mathrm{kg}^{-1}\right)$ & $765.2 \pm 126.3$ & $832.7 \pm 31.34$ & $771.9 \pm 76.3$ & $694.4 \pm 85.4$ & $0.88(+)$ & 16.61(-) \\
\hline
\end{tabular}

Table 9b. Soil Parameters Changes of the Second Year Cover-Cropping Practices in May-August 2019 on Site 7 $(+)$ : increase, (-): decrease, NA: no change

\begin{tabular}{|c|c|c|c|c|c|c|}
\hline \multirow[t]{2}{*}{ Soil Parameters } & \multicolumn{2}{|c|}{ Mean of Pre Soil Samples } & \multicolumn{2}{|c|}{ Mean of Post Soil Samples } & \multicolumn{2}{|l|}{ \% Change } \\
\hline & Fallow & Cover Crops & Fallow & Cover Crops & Fallow & Cover Crops \\
\hline BD $\left(\mathrm{g} \mathrm{cm}^{-3}\right)$ & 1.15 & $1.05 \pm 0.06$ & 1.12 & $1.05 \pm 0.11$ & $2.61(-)$ & NA \\
\hline MWHC (\%) & 72.04 & $76.9 \pm 6.72$ & 66.04 & $70.04 \pm 2.31$ & $8.33(-)$ & $8.92(-)$ \\
\hline pH & 8.09 & $7.73 \pm 0.12$ & 7.8 & $7.82 \pm 0.11$ & $3.58(-)$ & $1.16(+)$ \\
\hline OM (\%) & 11.4 & $11.1 \pm 1.67$ & 10 & $9.89 \pm 1.73$ & $12.28(-)$ & $10.9(-)$ \\
\hline Active C (mg kg-1) & 1286 & $1288 \pm 62$ & 937.4 & $904.1 \pm 67.5$ & $27.1(-)$ & $29.79(-)$ \\
\hline CEC $\left(\mathrm{cmolc} \mathrm{kg}^{-1}\right)$ & 16.47 & $15.93 \pm 2.72$ & 4.43 & $5.06 \pm 1.4$ & $73.1(-)$ & 68.24(-) \\
\hline TKN (mg kg-1) & 3521 & $3921 \pm 881$ & 1722 & $1366 \pm 280$ & $51.09(-)$ & $65.16(-)$ \\
\hline Total $P\left(\mathrm{mg} \mathrm{kg}^{-1}\right)$ & 949 & $961.3 \pm 260.1$ & 394 & $527.5 \pm 199$ & $58.48(-)$ & $45.13(-)$ \\
\hline $\operatorname{M3P}\left(\mathrm{mg} \mathrm{kg}^{-1}\right)$ & 280 & $189.1 \pm 44$ & 135.5 & $131.4 \pm 28.6$ & $51.61(-)$ & $30.52(-)$ \\
\hline $\operatorname{M3K}\left(\mathrm{mg} \mathrm{kg}^{-1}\right)$ & 244.7 & $43.84 \pm 21.68$ & 742.6 & $80.84 \pm 69.6$ & $203.47(+)$ & $84.4(+)$ \\
\hline Soil Protein $\left(\mathrm{mg} \mathrm{kg}^{-1}\right)$ & 630.9 & $674 \pm 40.6$ & 798.2 & $822.2 \pm 51$ & $26.52(+)$ & $21.98(+)$ \\
\hline
\end{tabular}

The farm in Site 8 performed summer cover-cropping practices with cowpea and sunn hemp mix in both 2018 and 2019. Compared to fallow, a higher decrease in MWHC, active C, and CEC was observed in cover crops over both summers of 2018 and 2019 (Table 10a and b). OM and soil protein were increased by performing cover-cropping practices. However, different percentage changes were observed in soil pH, M3P, and M3K in these two years. Soil pH and M3P increased in both cover crops and fallow in 2018 while they decreased in 2019. However, M3K presented an opposite trend of M3P.

Table 10a. Soil Parameters Changes of The First Year Cover-Cropping Practices in May-August 2018 on Site 8 $(+)$ increase, $(-)$ decrease

\begin{tabular}{|c|c|c|c|c|c|c|}
\hline \multirow[t]{2}{*}{ Soil Parameters } & \multicolumn{2}{|c|}{ Mean of Pre Soil Samples } & \multicolumn{2}{|c|}{ Mean of Post Soil Samples } & \multicolumn{2}{|c|}{ \% Change } \\
\hline & Fallow & Cover Crops & Fallow & Cover Crops & Fallow & Cover Crops \\
\hline BD $\left(\mathrm{g} \mathrm{cm}^{-3}\right)$ & $1.32 \pm 0.02$ & $1.43 \pm 0.02$ & $1.43 \pm 0.01$ & $1.48 \pm 0.03$ & $8.33(+)$ & $3.45(+)$ \\
\hline MWHC (\%) & $46.08 \pm 2.83$ & $44.08 \pm 3.27$ & $47.54 \pm 7.78$ & $38.29 \pm 1.71$ & $3.17(+)$ & 13.14(-) \\
\hline pH & $7.1 \pm 0.07$ & $6.79 \pm 0.1$ & $7.69 \pm 0.1$ & $7.04 \pm 0.21$ & $8.31(+)$ & $3.68(+)$ \\
\hline OM (\%) & 1.6 & $1.15 \pm 0.1$ & $1.49 \pm 0.14$ & $1.34 \pm 0.57$ & $6.88(-)$ & $16.52(+)$ \\
\hline Active $\mathbf{C}\left(\mathrm{mg} \mathrm{kg}^{-1}\right)$ & $1176 \pm 7.13$ & $1142 \pm 9.93$ & $292.3 \pm 89.6$ & $195.8 \pm 49.5$ & 75.14(-) & $82.84(-)$ \\
\hline CEC (cmolc kg-1) & $2.03 \pm 0.4$ & $1.62 \pm 0.24$ & $1.55 \pm 0.14$ & $1.22 \pm 0.31$ & $23.64(-)$ & $24.69(-)$ \\
\hline TKN (mg kg-1) & $786 \pm 77.9$ & $504.4 \pm 49.6$ & $599.3 \pm 192.4$ & $501.9 \pm 72.2$ & $23.76(-)$ & $0.5(-)$ \\
\hline Total $P\left(\mathrm{mg} \mathrm{kg}^{-1}\right)$ & $371 \pm 20.2$ & $461 \pm 60.5$ & $326.8 \pm 53.03$ & $446.1 \pm 101$ & $11.93(-)$ & $3.23(-)$ \\
\hline $\operatorname{M3P}\left(\mathrm{mg} \mathrm{kg}^{-1}\right)$ & $208.2 \pm 15.8$ & $308.5 \pm 40.7$ & $252.1 \pm 4.45$ & $334.5 \pm 40.3$ & $21.06(+)$ & $8.44(+)$ \\
\hline $\operatorname{M3K}\left(\mathrm{mg} \mathrm{kg}^{-1}\right)$ & $289.2 \pm 4.81$ & $192.5 \pm 70.4$ & $6.6 \pm 1.13$ & $20.5 \pm 9.89$ & $97.72(-)$ & $89.35(-)$ \\
\hline Soil Protein $\left(\mathrm{mg} \mathrm{kg}^{-1}\right)$ & $177.5 \pm 12.13$ & $114.6 \pm 21.1$ & $172.8 \pm 9.78$ & $151.7 \pm 2.8$ & $2.66(-)$ & $32.41(+)$ \\
\hline
\end{tabular}


Table 10b. Soil Parameters Changes of The Second Year Cover-Cropping Practices in May-August 2019 on Site $8(+)$ increase, (-) decrease

\begin{tabular}{|c|c|c|c|c|c|c|}
\hline \multirow[t]{2}{*}{ Soil Parameters } & \multicolumn{2}{|c|}{ Mean of Pre Soil Samples } & \multicolumn{2}{|c|}{ Mean of Post Soil Samples } & \multicolumn{2}{|l|}{$\%$ Change } \\
\hline & Fallow & Cover Crops & Fallow & Cover Crops & Fallow & Cover Crops \\
\hline BD $\left(\mathrm{g} \mathrm{cm}^{-3}\right)$ & $1.36 \pm 0.03$ & $1.41 \pm 0.03$ & $1.31 \pm 0.06$ & $1.38 \pm 0.05$ & $3.68(-)$ & $2.13(-)$ \\
\hline MWHC (\%) & 46.04 & $44.54 \pm 1.91$ & $45.04 \pm 4.24$ & $39.04 \pm 1.15$ & 2.1 & $12.35(-)$ \\
\hline pH & $7.48 \pm 0.03$ & $6.74 \pm 0.27$ & $6.54 \pm 0.69$ & $6.72 \pm 0.17$ & $12.57(-)$ & $0.3(-)$ \\
\hline OM $(\%)$ & $1.3 \pm 0.14$ & & $1.5 \pm 0.14$ & & $15.38(+)$ & $8.7(+)$ \\
\hline Active C (mg kg-1) & $308.9 \pm 1.02$ & $245.9 \pm$ & $313.9 \pm 36.7$ & $211.7 \pm$ & & $1(-)$ \\
\hline CEC $\left(\mathrm{cmolc} \mathrm{kg}^{-1}\right)$ & $1.93 \pm 0.12$ & $1.83 \pm 0.24$ & $0.67 \pm 0.02$ & $0.55 \pm 0.15$ & $65.28(-)$ & 69.95(-) \\
\hline TKN $\left(\mathrm{mg} \mathrm{kg}^{-1}\right)$ & $508.7 \pm 2$ & $420 \pm 53.12$ & $308 \pm 29.7$ & $274.8 \pm 26.4$ & $39.45(-)$ & $34.58(-)$ \\
\hline Total $\mathbf{P}\left(\mathrm{mg} \mathrm{kg}^{-1}\right)$ & $260.5 \pm 22.6$ & $469.6 \pm 140.4$ & $244.6 \pm 31.5$ & $300.3 \pm 45.8$ & $6.12(-)$ & $36.05(-)$ \\
\hline $\operatorname{M3P}\left(\mathrm{mg} \mathrm{kg}^{-1}\right)$ & $205.6 \pm 10.8$ & $297 \pm 35.6$ & $183.5 \pm 9.6$ & $212.6 \pm 50.5$ & $10.75(-)$ & $28.42(-)$ \\
\hline $\operatorname{M3K}\left(\mathrm{mg} \mathrm{kg}^{-1}\right)$ & $39.2 \pm 9.19$ & $37.8 \pm 8.32$ & $84.8 \pm 74.5$ & $91.4 \pm 28.1$ & $116.33(+)$ & $141.75(+)$ \\
\hline Soil Protein $\left(\mathrm{mg} \mathrm{kg}^{-1}\right)$ & $159.4 \pm 17.4$ & $143.5 \pm 7.2$ & $183.1 \pm 26.7$ & $179.6 \pm 30.9$ & $14.91(+)$ & $25.21(+)$ \\
\hline
\end{tabular}

The farm in Site 9 performed summer cover-cropping practice with sunn hemp in 2018. Compared to fallow, BD showed an increase due to cover-cropping practice (Table 11). All the soil properties except BD presented the similar percentage change trend between fallow and cover crops. Soil OM and soil protein showed a higher percentage increase in cover crops than in fallow while active C, CEC, TKN, and M3K showed a higher percentage decrease in cover crops.

Table 11. Soil Parameters Changes of The First Year Cover-Cropping Practices in May-August 2018 on Site 9

\begin{tabular}{|c|c|c|c|c|c|c|}
\hline \multirow[t]{2}{*}{ Soil Parameters } & \multicolumn{2}{|c|}{ Mean of Pre Soil Samples } & \multicolumn{2}{|c|}{ Mean of Post Soil Samples } & \multicolumn{2}{|c|}{ \% Change } \\
\hline & Fallow & Cover Crops & Fallow & Cover Crops & Fallow & Cover Crops \\
\hline BD $\left(\mathrm{g} \mathrm{cm}^{-3}\right)$ & 1.37 & $1.37 \pm 0.02$ & 1.35 & $1.48 \pm 0.02$ & $1.46(-)$ & $8.03(+)$ \\
\hline MWHC (\%) & 48.08 & $46.08 \pm 3.35$ & 51.04 & $46.87 \pm 3.13$ & $6.16(+)$ & $1.71(+)$ \\
\hline pH & 7.78 & $6.68 \pm 0.66$ & 7.48 & $6.55 \pm 0.26$ & $3.86(-)$ & $1.95(-)$ \\
\hline OM $(\%)$ & 3 & $1.93 \pm 0.48$ & 3.19 & $2.06 \pm 0.39$ & $6.33(+)$ & $6.74(+)$ \\
\hline Active C (mg kg $\left.{ }^{-1}\right)$ & 1230 & $1208 \pm 22.94$ & 571.7 & $489.4 \pm 75.57$ & $53.51(-)$ & $59.5(-)$ \\
\hline CEC (cmolc kg-1) & 5.02 & $3.02 \pm 1.06$ & 3.72 & $1.84 \pm 0.5$ & $25.9(-)$ & $39.07(-)$ \\
\hline TKN $\left(\mathrm{mg} \mathrm{kg}^{-1}\right)$ & 1152.2 & $792.7 \pm 164.2$ & 1150 & $692.8 \pm 70.91$ & $1.79(-)$ & 12.61(-) \\
\hline Total $\mathbf{P}\left(\mathrm{mg} \mathrm{kg}^{-1}\right)$ & 473.3 & $168.8 \pm 149$ & 581.8 & $171.9 \pm 102.4$ & $22.93(+)$ & $1.82(+)$ \\
\hline $\operatorname{M3P}\left(\mathbf{m g ~ k g}^{-1}\right)$ & 254.4 & $95.94 \pm 61.71$ & 196.7 & $89.83 \pm 66.45$ & $22.68(-)$ & $6.37(-)$ \\
\hline $\operatorname{M3K}\left(\mathrm{mg} \mathrm{kg}^{-1}\right)$ & 134.1 & $61.43 \pm 33.98$ & 38 & $9.17 \pm 6.4$ & $71.66(-)$ & $85.07(-)$ \\
\hline Soil Protein $\left(\mathrm{mg} \mathrm{kg}^{-1}\right)$ & 334.4 & $325.9 \pm 63.67$ & 344.6 & $361.4 \pm 63.1$ & $3.03(+)$ & $10.91(+)$ \\
\hline
\end{tabular}

The farm in Site 10 performed summer cover-cropping practice with sunn hemp in 2019. Compared to fallow, BD showed an increase due to cover-cropping practice (Table 12). However, there was a decrease in MWHC, pH, $\mathrm{OM}$, active $\mathrm{C}, \mathrm{CEC}, \mathrm{TKN}$, and M3P in cover-cropping plots.

Table 12. Soil Parameters Changes of The First Year Cover-Cropping Practices in May-August 2019 on Site 10

\begin{tabular}{|c|c|c|c|c|c|c|}
\hline \multirow[t]{2}{*}{ Soil Parameters } & \multicolumn{2}{|c|}{ Mean of Pre Soil Samples } & \multicolumn{2}{|c|}{ Mean of Post Soil Samples } & \multicolumn{2}{|l|}{ \% Change } \\
\hline & Fallow & Cover Crops & Fallow & Cover Crops & Fallow & Cover Crops \\
\hline $\mathrm{BD}\left(\mathrm{g} \mathrm{cm}^{-3}\right)$ & 1.44 & $1.39 \pm 0.08$ & 1.43 & $1.46 \pm 0.07$ & $0.69(-)$ & $5.04(+)$ \\
\hline MWHC (\%) & 40.04 & $45.71 \pm 3.2$ & 40.04 & $39.71 \pm 4.08$ & NA & $13.13(-)$ \\
\hline pH & 6.52 & $6.4 \pm 0.26$ & 7.32 & $6.27 \pm 0.32$ & $12.27(+)$ & $2.03(-)$ \\
\hline OM $(\%)$ & 1.8 & $2.43 \pm 0.57$ & 2.8 & $2.27 \pm 0.55$ & $55.56(+)$ & $6.58(-)$ \\
\hline Active $\mathbf{C}\left(\mathrm{mg} \mathrm{kg}^{-1}\right)$ & 472.3 & $619 \pm 94$ & 473.8 & $427.7 \pm 89.8$ & $0.3(+)$ & $30.9(-)$ \\
\hline CEC $\left(\right.$ cmolc kg $\left.^{-1}\right)$ & 3.93 & $4.19 \pm 1.02$ & 1.76 & $1.01 \pm 0.36$ & $55.22(-)$ & $75.82(-)$ \\
\hline TKN (mg kg $\left.{ }^{-1}\right)$ & 689.5 & $775.1 \pm 197.5$ & 427 & $353.5 \pm 167.1$ & $38.06(-)$ & $54.39(-)$ \\
\hline Total $P\left(\mathrm{mg} \mathrm{kg}^{-1}\right)$ & 283 & $199.4 \pm 57.6$ & 297.5 & $216.2 \pm 132.9$ & $5.12(+)$ & $8.41(+)$ \\
\hline $\operatorname{M3P}\left(\mathrm{mg} \mathrm{kg}^{-1}\right)$ & 194.6 & $115.3 \pm 13.7$ & 211.8 & $105 \pm 31.7$ & $8.84(+)$ & $8.95(-)$ \\
\hline $\operatorname{M3K}\left(\mathrm{mg} \mathrm{kg}^{-1}\right)$ & 53.7 & $30.18 \pm 7.06$ & 143.5 & $48.12 \pm 10.16$ & $167.23(+)$ & $59.44(+)$ \\
\hline Soil Protein $\left(\mathrm{mg} \mathrm{kg}^{-1}\right)$ & 207.9 & $312.6 \pm 51.3$ & 374.9 & $396.6 \pm 83.7$ & $80.29(+)$ & $26.88(+)$ \\
\hline
\end{tabular}

\subsection{Effects of Cover Crops on All Farms}

When considering cover-cropping practice as a whole, regardless of different sites, climates and cover crops species planted on their farms, it showed effects on various soil parameters including BD, MWHC, OM, CEC, soil protein, TKN, and M3K. There were no significant differences among pre and post soil samples collected in these two years from fallow fields (Figure 3 II, III, IV, VI, VII, VIII and XI). However, there was a significant 
increase in $\mathrm{OM}$ in the second year of performing cover-cropping practice, which subsequently resulted in a significantly higher MWHC and lower BD (Figure 3 II, III, and IV). There was a significantly higher CEC in the pre soil samples while a significantly higher level of soil protein in the post soil samples was observed in the second year of planting cover crops (Figure 3 VI and VII). Compared to fallow, cover crops tended to decrease the soil TKN level since the TKN was lower after performing cover-cropping practice in both year 1 and year 2 , particularly a significant lower TKN was observed in the second year (Figure $3 \mathrm{VIII})$. In the case of M3K, there was a significant decrease in the post soil samples in year 1 and pre soil samples in year 2 (Figure 3 XI). In terms of soil $\mathrm{pH}, \mathrm{TP}$, and M3P, there were no significant changes in all soil samples collected from both fallow and cover crops fields in these two years. Active $\mathrm{C}$ decreased in fallow fields over time while a significant increase was observed in pre soil samples collected in the second year of performing cover-cropping practice compared to the post soil samples collected in the first year.
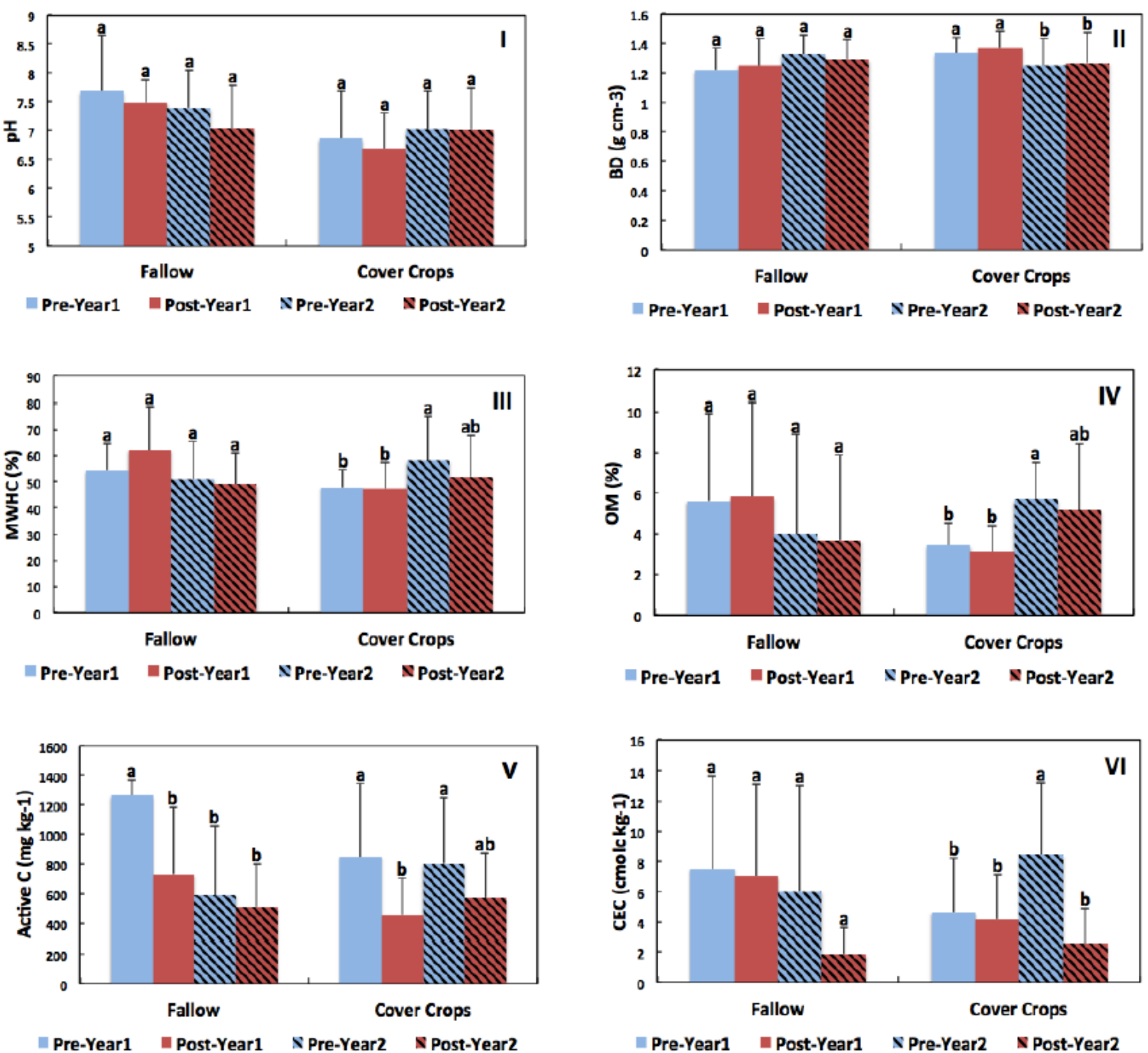

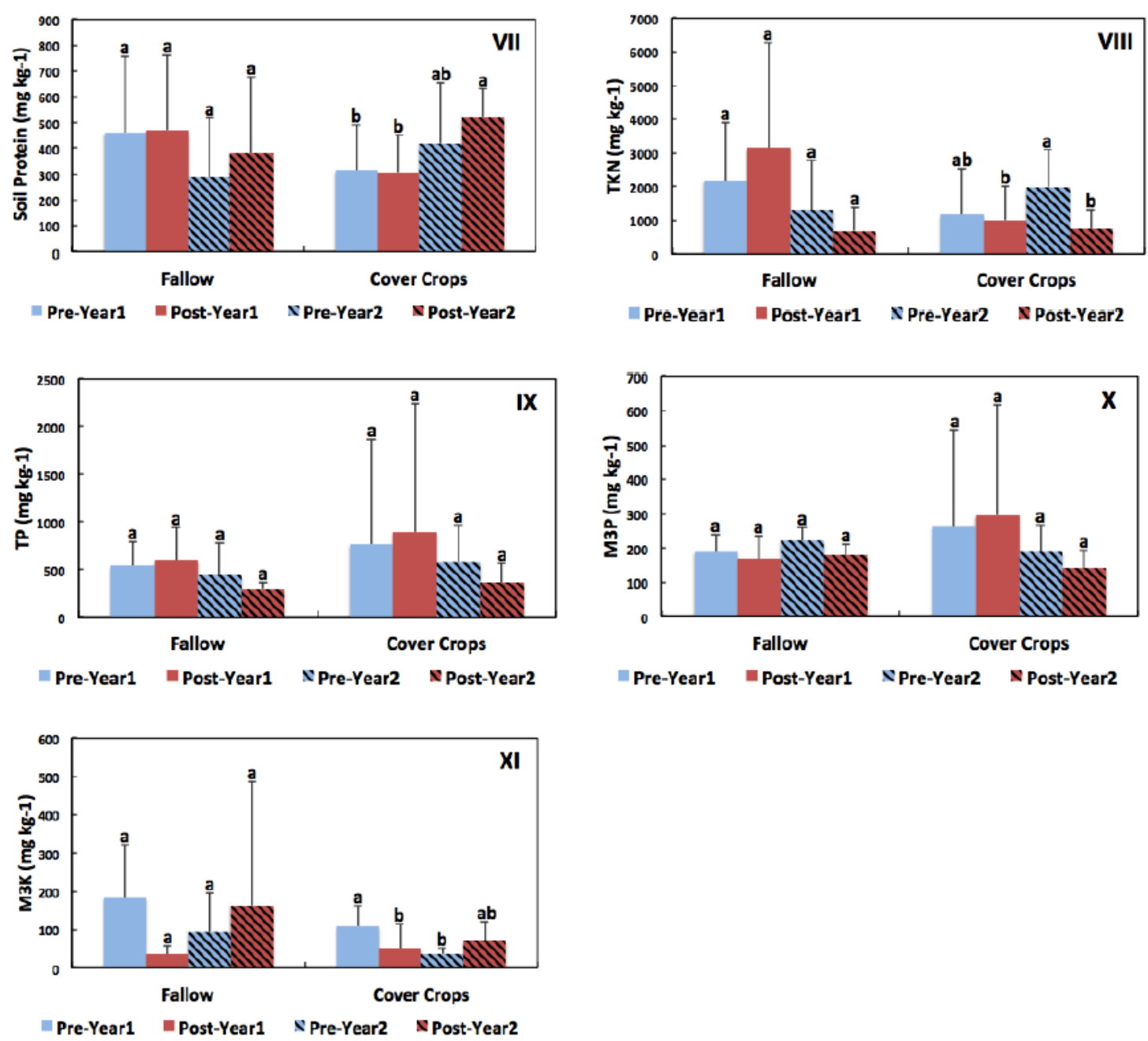

Figure 3 (I-XI). Changes in soil parameters pre and post soil samples between fallow and cover-cropping practice from all farms in 2018-2019 (mean and standard deviation). Means followed by different lower case letters within Fallow/Cover Crops are significantly different ( $\mathrm{p}<0.05)$

Pre-Year 1= Soil samples collected before fallow/cover-cropping practice in 2018

Post-Year 1= Soil samples collected right after cover crops termination in 2018

Pre-Year 2= Soil samples collected before fallow/cover-cropping practice in 2019

Post-Year 2= Soil samples collected right after cover crops termination in 2019

\section{Discussion}

\subsection{Evidence from the Individual farm Results}

Overall, differences due to cover-cropping practice in our study were site specific because of the various cover crops species, soil types, climates, cropping systems, and managements on their farms. Specifically, three farms showed a decrease in BD at the first year of planting cover crops while five farms showed an increase. Two farms showed no change by the end of year 2 due to cover-cropping while site 8 showed a decrease and site 10 presented an increase (Table 13). Typically, lowering soil BD is favored since it is beneficial for root growth, aeration and water movement, and alleviates soil compaction (Xu, Bhadha, Rabbany \& Swanson, 2019). Soil pH was reduced in seven farms, whereas two farms showed an increase after the first year of planting cover crops. It seems cover crops tended to decrease soil $\mathrm{pH}$. Five farms showed an increase in OM after the first year of cover-cropping, of which four of them planted mixes of different cover crops. It might indicate that cover crop mixtures were more likely to increase OM because the mix produced more biomass than pure stands (Elhakeem et al., 2019). Five farms showed an increase in MWHC while the other four farms presented a decrease after the 
first year of planting cover crops. All farms that planted the second year of cover crops presented a decrease in MWHC. The increase in MWHC might be because of the increase in OM content in topsoil. Active C represents a soil labile carbon pool that microbial communities can easily mineralize (Bongiorno et al., 2019). The farms that performed winter cover-cropping practices (Site 1 to 4 ) showed an increase in active $\mathrm{C}$ after termination while those that planted summer cover crops (Site 5 to 10) showed a decrease. This result might indicate that the cover-cropping practice performed in our study did not show clear positive/negative effects on active C. It seems active $\mathrm{C}$ is more related to climate factors such as temperature since it showed seasonal variations. The labile carbon pool was reported as the most sensitive since it was easily affected by fluctuation in environmental conditions (Sahoo et al., 2019). However, the seasonal dynamics of active $\mathrm{C}$ is less known currently according to Bongiorno et al. (2019). CEC is primarily controlled by organic matter content and the fraction of clay-size particles in soils (Parfitt, Giltrap, \& Whitton, 1995). The soils of the ten sites (except Site 7) in our study region are sandy texture that are dominated with coarse particles, inherently have low soil OM content $(<5 \%)$ and low CEC values $\left(<10 \mathrm{cmolc} \mathrm{kg}^{-1}\right)$. Site 7 is an organic farm with years of OM inputs, thus has a relatively higher soil OM $(>10 \%)$ and CEC $\left(>10 \mathrm{cmolc} \mathrm{kg}^{-1}\right)$. In our study, four farms showed an increase in CEC after growing cover crops while the remaining six sites presented a decrease. The increase in CEC might be due to the OM inputs from cover crops. In the case of TKN, seven farms showed a decrease after cover crops, even for those (such as Site 8 to 10) that grew legume cover crops. This result might indicate that non-legume cover crops such as those grown in Site 2 to 4 scavenged or trapped soil nitrate that would otherwise be subject to losses by leaching or denitrification (Kladivko, 2016). The legume cover crops that were supposed to increase soil $\mathrm{N}$ level such as on Site 8 to 9, no increase in TKN was observed as well. However, although the TKN showed a decrease trend after cover-cropping practices in most farms, soil protein level in seven farms showed an increase. Soil protein represents the amount of organically bound $\mathrm{N}$ in soil $\mathrm{OM}$ that microbial communities can mineralize (Schindelbeck, Moebius-Clune, Moebius-Clune, Kurtz, \& van Es, 2016). Our result indicated that cover crops helped to build up soil protein level in sandy soils, which is a positive change since preserving microbially degradable $\mathrm{N}$ is good to provide subsequent cash crops with its $\mathrm{N}$ needs over time. In terms of $\mathrm{P}$, six farms showed an increase in Total $\mathrm{P}$ and seven farms presented an increase in M3P. From a nutrient perspective, this is a good sign as it indicated larger amounts of $\mathrm{P}$ would be available for subsequent cash crops that are being planted. In the case of K, only one farm presented an increase in Year 1 while three farms showed an increase in Year 2. In general, M3K tended to decrease in cover-cropping fields, which might indicate that supplementary $\mathrm{K}$ is required prior to planting subsequent cash crops. 
Table 13. Soil Parameters Percentage Changes for all experimental sites over 2018 and 2019 (+) increase, (-) decrease, NA no change

\begin{tabular}{|c|c|c|c|c|c|c|c|c|c|c|c|c|}
\hline & Site & Site & Site & Site & Site & Site & \multicolumn{2}{|l|}{ Site } & \multicolumn{2}{|l|}{ Site } & Site & Site \\
\hline & 1 & 2 & 3 & 4 & 5 & 6 & & \multicolumn{2}{|l|}{8} & 9 & 10 \\
\hline & $\begin{array}{l}\text { Various } \\
\text { legumes } \\
\text { and } \\
\text { grains } \\
\text { mix }\end{array}$ & $\begin{array}{l}\text { Oats } \\
\text { and rye } \\
\text { mix }\end{array}$ & Oats & Oats & $\begin{array}{l}\text { Buckwheat } \\
\text { followed by } \\
\text { cowpea and sunn } \\
\text { hemp mix }\end{array}$ & $\begin{array}{l}\text { Sunn } \\
\text { hemp }\end{array}$ & \multicolumn{2}{|c|}{$\begin{array}{l}\text { Cowpea and } \\
\text { sudan grass } \\
\text { mix, } \\
\text { Cowpea and } \\
\text { sunn hemp } \\
\text { mix Cowpea } \\
\text { only }\end{array}$} & \multicolumn{2}{|c|}{$\begin{array}{l}\text { Cowpea and } \\
\text { sunn hemp mix }\end{array}$} & $\begin{array}{l}\text { Sunn } \\
\text { hemp }\end{array}$ & $\begin{array}{l}\text { Sunn } \\
\text { hemp }\end{array}$ \\
\hline & \multicolumn{4}{|l|}{ Ultisols } & Entisols & \multicolumn{5}{|l|}{ Alfisols } & \multicolumn{2}{|c|}{ Spodosols } \\
\hline & $\begin{array}{l}\text { Year } \\
1,2\end{array}$ & $\begin{array}{l}\text { Year } \\
1\end{array}$ & $\begin{array}{l}\text { Year } \\
1\end{array}$ & $\begin{array}{l}\text { Year } \\
1\end{array}$ & Year 1 & Year 1 & $\begin{array}{l}\text { Year } \\
1\end{array}$ & $\begin{array}{l}\text { Year } \\
2\end{array}$ & $\begin{array}{l}\text { Year } \\
1\end{array}$ & $\begin{array}{l}\text { Year } \\
2\end{array}$ & Year 1 & Year 2 \\
\hline $\begin{array}{l}\mathrm{BD} \\
\left.\mathrm{cm}^{-3}\right)\end{array}$ & NA & - & + & + & - & - & + & NA & + & - & + & + \\
\hline $\begin{array}{l}\text { MWHC } \\
(\%)\end{array}$ &,+- & - & - & - & + & + & + & - & - & - & + & - \\
\hline $\mathrm{pH}$ &,+- & - & - & - & - & - & - & + & + & - & - & - \\
\hline $\mathrm{OM}(\%)$ &,+- & + & - & - & + & - & - & - & + & + & + & - \\
\hline $\begin{array}{l}\text { Active C } \\
\left(\mathrm{mg} \mathrm{kg}^{-1}\right)\end{array}$ &,-+ & + & + & + & - & - & - & - & - & - & - & - \\
\hline $\begin{array}{l}\text { CEC } \\
\left(\mathrm{cmolc}^{-1}\right. \\
\left.\mathrm{kg}^{-1}\right)\end{array}$ &,-+ & + & - & - & + & + & - & - & - & - & - & - \\
\hline$\underset{\left.\mathrm{kg}^{-1}\right)}{\mathrm{TKN}} \quad(\mathrm{mg}$ &,+- & - & - & - & + & + & - & - & - & - & - & - \\
\hline $\begin{array}{l}\text { Total P } \\
\left(\mathrm{mg} \mathrm{kg}^{-1}\right)\end{array}$ &,+- & + & - & + & + & - & - & - & - & - & + & + \\
\hline $\begin{array}{l}\text { M3P } \quad(\mathrm{mg} \\
\left.\mathrm{kg}^{-1}\right)\end{array}$ &,-+ & + & - & + & + & + & + & - & + & - & - & - \\
\hline $\begin{array}{l}\text { M3K (mg } \\
\left.\mathrm{kg}^{-1}\right)\end{array}$ &,-- & - & - & + & - & - & - & + & - & + & - & + \\
\hline $\begin{array}{l}\text { Soil } \\
\text { Protein } \\
\left(\mathrm{mg} \mathrm{kg}^{-1}\right)\end{array}$ &,+- & - & - & - & + & + & - & + & + & + & + & + \\
\hline
\end{tabular}

\subsection{Evidence from All Farms Results}

Cover-cropping practice could be a promising option for Florida's growers to replace their fallow fields since it generally statistically showed soil health benefits across all the farms. Compared to fallow, soil OM, BD, MWHC, and soil protein showed a significant increase in cover crop fields in the second year, which presented a positive change towards building up soil health. Typically, producing approximately 5 tons dry matter/acre equals to raising organic matter by $1 \%$ in the top 6-inch layer of sandy soils ( $\mathrm{Li}$ et al., 2006). We observed approximately $2 \%$ significant increase in soil OM after one year of cover crop practice in our study. The additional OM is especially critical for most Florida's soils since they are generally of sandy texture with low nutrient and water retention capacity and easily prone to nutrient leaching. Increased water holding capacity allows more water to be stored in the soil for plant uptake. Reduced bulk density helps to alleviate soil compaction. The most positive effect observed in our study is soil protein level was enhanced in the cover crop fields by the end of second year. Soil protein represents the large bioavailable $\mathrm{N}$ pool in soils for microbial communities. The increase in soil protein indicated that cover crops assisted in optimizing $\mathrm{N}$ cycling in soils that slowly release available $\mathrm{N}$ for subsequent cash crops over time, thus reducing soluble forms of $\mathrm{N}$ losses (Kladivko, 2016; Schindelbeck, B.N. Moebius-Clune, D.J. Moebius-Clune, Kurtz, \& van Es, 2016). Although soil TKN level was significantly decreased due to cover crops, the increase of soil protein over time is a good sign. Moreover, reduction of TKN may be beneficial from the environmental perspective since no excessive $\mathrm{N}$ was added to the soils. In the case of $\mathrm{K}$, the soil M3K level also showed a significant decrease trend due to cover-cropping practice, which indicated that cover crops might deplete soil $\mathrm{K}$ or no obvious effects of cover crops on reserving $\mathrm{K}$ in sandy soils was observed in our study. In terms of soil $\mathrm{pH}$, although it seems cover crops tend to decrease soil $\mathrm{pH}$ in most farms, there were no significant changes. Similarly, total P and M3P also presented an increase in most farms, however no significant changes were found. Active $\mathrm{C}$ showed a significant increase in cover crops fields in the second year, which might indicate that cover crops helped to build up active $\mathrm{C}$ level in the soil. Both active $\mathrm{C}$ and soil protein increase provided evidence that cultivating cover crops could 
be a beneficial practice to enhance soil microbial quality such as abundance, activity, and diversity (Balota, Calegari, Nakatani, \& Coyne, 2014; Kim, Zabaloy, Guan, \& Villamil, 2020).

Although cover crops could be a beneficial practice to maintain and improve soil health, the decision of whether or not it can be successfully integrated on farms depends on many factors. As shown in individual farm results in our study, the cover crops performance varied in different farms due to different cover crop species, climates, soil types, cropping systems and management. Incorporating cover crops into a cropping system requires time, money, inputs, labor, machinery, and related cover crop management information (Kaspar and Singer, 2011).

\section{Conclusions}

In general, cultivating cover crops over fallow periods could be a viable option for Florida's growers since cover crops showed beneficial effects on soil health over all the farms statistically. Compared to fallow, soil OM, BD, MWHC, and soil protein showed increases in cover crop fields of most farms, which presented a positive change towards building up soil health. Although soil TKN level was significantly decreased due to cover crops, soil protein level building up over time was the most positive change for soil health. M3K decreased in cover-crop fields, which indicated that supplementary $\mathrm{K}$ was required prior to planting subsequent cash crops. Cover crops performance depends on many factors such as cover crop species selection, climate conditions, soil types, cropping systems, and managements. Moreover, implementing cover crops into a cropping system requires time, money, inputs, labor, machinery, and related cover crop management information. These issues are also limiting factors for growers to adopt cover-cropping practice on their farms. Therefore, further studies, particularly long-term studies on overcoming these supplies, management, and service problems should be considered.

\section{Acknowledgments}

This study was funded by the USDA - Southern Sustainable Agriculture Research and Education (OS18-114), and USDA National Institute of Food and Agriculture - Hatch Project FLA-ERC-005552. We would like to thank our collaborative growers, including Patrick Troy, Kim Yates, Tripp Whidden (R.C. Hatton Farms), Derek Orsinego (GMI Farm), Margaret Duriez (Lox farms), Noah Shitama (Swallowtail Farm), Libby Schmidt (Spring Head Ranch), and Jeff Willis (Columbia Livestock Market). Mr. Salvador Galindo and Martino Trotta for assistance with field and lab work.

\section{References}

Balota, E. L., Calegari, A., Nakatani, A. S., \& Coyne, M. S. (2014). Benefits of winter cover crops and no-tillage for microbial parameters in a Brazilian Oxisol: A long-term study. Agriculture, ecosystems \& environment, 197, 31-40. https://doi.org/10.1016/j.agee.2014.07.010

Bhadha, J. H., Capasso, J., Khatiwada, R., Swanson, S. \& Laborde, C. (2017a). Raising soil organic matter content to improve water holding capacity. UF/IFAS Extension Publication \# SL447. https://doi.org/10.32473/edis-ss661-2017

Bhadha, J. H., Capasso, J., Schindelbeck, R. S., \& Bacon, A. (2017b). Tools for evaluating soil health. UF/IFAS Extension Publication \# SL443. https://doi.org/10.32473/edis-ss657-2017

Bhadha, J. H., Wright, A. L., \& Snyder, G. H. (2020). Everglades Agricultural Area aoil subsidence and sustainability. UF/IFAS Extension Publication \# SL311. https://doi.org/10.32473/edis-ss523-2020

Bongiorno, G., Bünemann, E. K., Oguejiofor, C. U., Meier, J., Gort, G., Comans, R., Mäder, P., Brussaard, L., \& de Goede, R. (2019). Sensitivity of labile carbon fractions to tillage and organic matter management and their potential as comprehensive soil quality indicators across pedoclimatic conditions in Europe. Ecological Indicators, 99, 38-50. https://doi.org/10.1016/j.ecolind.2018.12.008

Dimitri, C., Effland, A., \& Conklin, N. (2005). The 20th century transformation of U.S. agriculture and farm policy. USDA. Economic Information Bulletin Number 3. Retrieved from www.ers.usda.gov

Elhakeem, A., van der Werf, W., Ajal, J., Lucà, D., Claus, S., Vico, R. A., \& Bastiaans, L. (2019). Cover crop mixtures result in a positive net biodiversity effect irrespective of seeding configuration. Agriculture, Ecosystems \& Environment, 285, 106627. https://doi.org/10.1016/j.agee.2019.106627

Fageria, N. K., Baligar, V. C., \& Clark, R. B. (2002). Micronutrients in crop production. Advances in Agronomy, 77, 185-268. https://doi.org/10.1016/S0065-2113(02)77015-6

Fischer, G., Shah, M., Tubiello, F. N., \& van Velhuizen, H. (2005). Socio-economic and climate change impacts on agriculture: an integrated assessment, 1990-2080. Philos Trans R Soc Lond B Biol Sci., 360, 2067-2083. https://doi.org/10.1098/rstb.2005.1744 
He, Z., Yang, X., Kahn, B. A., Stoffella, P. J., Calvert, D. V. (2000). Plant nutrition benefits of phosphorus, potassium, calcium, magnesium, and micronutrients from compost utilization. In Stoffella \& Kahn (Eds.), Compost utilization in horticultural cropping systems (pp. 307-320). Lewis Publishers, Boca raton, Florida.

Jenkinson, D. S., \& Powlson, D. S. (1976). The effects of biocidal treatments on metabolism in soil-V: A method for measuring soil biomass. Soil Biology and Biochemistry, 8(3), 209-213. https://doi.org/10.1016/0038-0717(76)90005-5

Kaspar, T. C., \& Singer, J. W. (2011). The use of cover crops to manage soil. In J. L. Hatfield \& T. J. Sauer, (Eds.), Soil Management: Building a stable base for agriculture. Madison (pp. 321-337). WI: American Society of Agronomy and Soil Science Society of America. https://doi.org/10.2136/2011.soilmanagement.c21

Kim, N., Zabaloy, M. C., Guan, K., \& Villamil, M. B. (2020). Do cover crops benefit soil microbiome? A meta-analysis of current research. Soil Biology and Biochemistry, 142, 107701. https://doi.org/10.1016/j.soilbio.2019.107701

Kladivko, E. (2016). Cover Crops for Soil Nitrogen Cycling. Retrieved from http://www.ag.purdue.edu/agry/extension/Documents/CoverCropsNitrogen.pdf

Li, Y. C., Hanlon, E. A., Klassen, W., Wang, Q., Olczyk, T., \& Ezenwa, I. V. (2006). Cover crop benefits for South Florida commercial vegetable producers. University of Florida, IFAS EDIS Publication \# SL-242.

Mylavarapu, R., Harris, W., \& Hochmuth, G. (2019). Agricultural soils of Florida. University of Florida, IFAS Extension Publication \# SL441.

Parfitt, R. L., Giltrap, D. J., \& Whitton, J. S. (1995). Contribution of organic matter and clay minerals to the cation exchange capacity of soils. Communications in Soil Science and Plant Analysis, 26(9-10), 1343-1355. https://doi.org/10.1080/00103629509369376

Sahoo, U. K., Singh, S. L., Gogoi, A., Kenye, A., \& Sahoo, S. S. (2019). Active and passive soil organic carbon pools as affected by different land use types in Mizoram, Northeast India. PloS one, 14(7), e0219969. https://doi.org/10.1371/journal.pone.0219969

Schindelbeck, R. R., Moebius-Clune, B. N., Moebius-Clune, D. J., Kurtz, K. S., \& van Es, H. M. (2016). Cornell University comprehensive assessment of soil health laboratory standard operating procedures. Cornell Univ., Ithaca, NY.

Snapp, S. S., Swinton, S. N., Labarta, R., Mutch, D., Black, J. R., Leep, Nyiraneza, J., \& O’Neil, K. (2005). Evaluating cover crops for benefits, costs and performance within cropping system niches. Agronomy Journal, 97, 322-332.

Sumner, M. E., \& Miller M. P. (1996). Cation exchange capacity and exchange coefficient. In Sparks (Ed.). Methods of soil analysis (pp. 1201-1230). part 3: Chemical Methods., Madison, Wisconsin. https://doi.org/10.2136/sssabookser5.3.c40

Treadwell, D., Klassen, W., \& Alligood, M. (2008). Annual cover crops in Florida vegetable systems Part 1. Objectives: Why grow cover crops? University of Florida, IFAS Extension Publication \# HS-387.

Xu, N., Bhadha, J. H., Rabbany, A., \& Swanson, S. (2019). Soil Health Assessment of Two Regenerative Farming Practices on Sandy Soils. Sustainable Agriculture Research, 8(526-2020-557), 61-71. https://doi.org/10.5539/sar.v8n4p61

\section{Copyrights}

Copyright for this article is retained by the author(s), with first publication rights granted to the journal.

This is an open-access article distributed under the terms and conditions of the Creative Commons Attribution license (http://creativecommons.org/licenses/by/3.0/). 